\title{
Rapidly recomputable EEG forward models for realistic head shapes
}

\author{
John J Ermer ${ }^{1,2}$, John C Mosher ${ }^{3}$, Sylvain Baillet ${ }^{4}$ and \\ Richard M Leahy ${ }^{5,6}$ \\ ${ }^{1}$ Signal \& Image Processing Institute, University of Southern California, Los Angeles, \\ CA 90089-2564, USA \\ 2 Raytheon Systems Company, El Segundo, CA 90245, USA \\ ${ }^{3}$ Los Alamos National Laboratory, Los Alamos, NM 87545, USA \\ ${ }^{4}$ Neurosciences Cognitives \& Imagerie Cerebrale CNRS UPR640_LENA, \\ Hopital de la Salpetriere, Paris, France \\ 5 Signal \& Image Processing Institute, University of Southern California, Los Angeles, \\ CA 90089-2564, USA \\ E-mail: ermer@sipi.usc.edu, mosher@lanl.gov, sylvain.baillet@chups.jussieu.fr and \\ leahy@sipi.usc.edu
}

Received 17 November 2000

\begin{abstract}
With the increasing availability of surface extraction techniques for magnetic resonance and $\mathrm{x}$-ray computed tomography images, realistic head models can be readily generated as forward models in the analysis of electroencephalography (EEG) and magnetoencephalography (MEG) data. Inverse analysis of this data, however, requires that the forward model be computationally efficient. We propose two methods for approximating the EEG forward model using realistic head shapes. The 'sensor-fitted sphere' approach fits a multilayer sphere individually to each sensor, and the 'three-dimensional interpolation' scheme interpolates using a grid on which a numerical boundary element method (BEM) solution has been precomputed. We have characterized the performance of each method in terms of magnitude and subspace error metrics, as well as computational and memory requirements. We have also made direct performance comparisons with traditional spherical models. The approximation provided by the interpolative scheme had an accuracy nearly identical to full BEM, even within $3 \mathrm{~mm}$ of the inner skull surface. Forward model computation during inverse procedures was approximately 30 times faster than for a traditional three-shell spherical model. Cast in this framework, high-fidelity numerical solutions currently viewed as computationally prohibitive for solving the inverse problem (e.g. linear Galerkin BEM) can be rapidly recomputed in a highly efficient manner. The sensor-fitting method has a similar one-time cost to the BEM method, and while it produces some improvement over a standard three-shell sphere, its performance does not approach that of the interpolation method. In both methods, there is a one-time cost associated with precomputing the forward solution over a set of grid points.
\end{abstract}

6 Author to whom correspondence should be addressed. 


\section{Introduction}

The objective of EEG inverse methods is to estimate neural current source characteristics given an observed set of noise-corrupted scalp-potential measurements. Solution of this EEG source localization or inverse problem typically requires a significant number of forward model evaluations. Nonlinear directed searches such as least-squares (Wang et al 1992) and MUSIC (Mosher et al 1992, Mosher and Leahy 1999) can require evaluation of the forward model at thousands of possible source locations. Because of their simplicity, ease of computation and relatively good accuracy, multilayer spherical models (Berg and Scherg 1994, Brody et al 1973, Zhang 1995) have traditionally been used for approximating the human head. The spherical model, however, does have several key drawbacks.

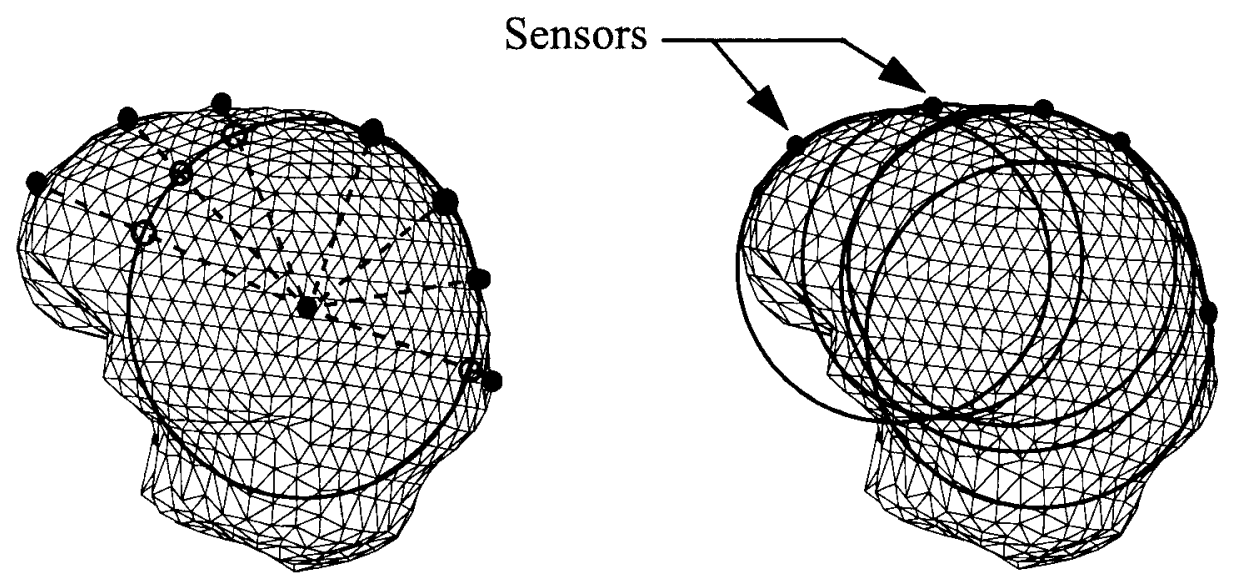

Figure 1. (Left) Spatial distortion of true sensor positions $(\mathbf{O})$ due to radial projection onto best-fit single-sphere model $(\mathrm{O})$. (Right) Schematic plot of sensor-weighted spheres model.

By its very shape, the spherical model distorts the true distribution of passive currents in the brain, skull and scalp. Spherical models also require that the sensor positions be projected onto the fitted sphere (figure 1) resulting in a distortion of the true sensor-dipole spatial geometry and ultimately the computed surface potential. The use of a single best-fitted multilayer sphere has the added drawback of incomplete coverage of the inner skull region, often ignoring areas such as the frontal cortex. In practice, this problem is typically resolved by fitting additional spheres to those regions not covered by the primary sphere. The use of these additional spheres results in added complication in the EEG forward model, since a neural source may be simultaneously inside of some spheres and outside others.

Using high-resolution spatial information obtained from x-ray computed tomography (CT) or magnetic resonance (MR) images, we can generate a more realistic head model. We represent the head as a set of contiguous regions bounded by surface tessellations of the scalp, outer skull and inner skull boundaries. Since accurate in vivo determination of internal conductivities is not currently possible, we assume that the conductivities are homogeneous and isotropic within each region. With the exception of simple geometries (e.g. spheres, ellipsoids), analytical solutions for the potentials over multilayer surfaces do not exist. For a surface of arbitrary shape, the surface potential can be found using the boundary element method (BEM) or other related techniques to solve the surface integral equations.

The major drawback of BEM and other numerical techniques is their computational cost, which can exceed that of multilayer sphere models by two or three orders of magnitude. In the 
past, the use of BEM was also limited by the large memory requirements for matrix inversion and the lack of reliable surface extraction methodologies. These problems are being overcome with the availability of high-density low-cost memory and improvements in surface extraction methodologies. Currently, lack of computationally efficient EEG forward modelling solutions for non-spherical surfaces appears to be the major barrier to widespread adoption of these more realistic head models.

Past work in the area of computationally efficient EEG forward models has primarily focused on multilayer spherical models, most notably in the work of Berg and Scherg (1994), deMunck and Peters (1993) and Zhang (1995). For realistic head models, Huang et al (1999) present a sensor-fitted sphere method for MEG whose accuracy approaches that of BEM with a computational cost on the order of a multilayer sphere. Here we describe an extension of this method to the forward EEG problem and a second method based on interpolation of a precomputed BEM solution. We show that the quality of the forward modelling solution approaches that of BEM, with a recomputation time approximately 30 times faster than that of a multilayer spherical model. The proposed methodology has the added benefit of providing whole-head coverage.

The three-dimensional forward-field interpolation methodology described here represents an extension to methods previously presented by Ermer et al (2000), and algorithms currently implemented as part of the 'BrainStorm' neuroimaging software package (Baillet et al 2000). We note also the recent publication by Yvert et al (2000), who describe a similar methodology with results consistent with ours.

The layout of this paper is as follows. Section 2 provides the basic definitions and an overview of the popular EEG forward models. A description of the proposed methods are presented in section 3 . Results and algorithm computational/memory requirements are presented in section 4. Final conclusions are drawn in section 5. The notation throughout this paper is as follows: in general, an italicized plain font is used to denote scalar quantities and boldface is used to indicate vectors and matrices. A superscript ' $T$ ' is used to denote the transpose operator.

\section{Background}

\subsection{The forward model}

The forward model relates a current dipole of moment $\boldsymbol{q}$ at location $\boldsymbol{r}_{\boldsymbol{q}}$ to the surface potential $v(r)$ at location $r$. Under the assumption that the head is represented as a multilayer surface composed of non-intersecting homogeneous 'shells' of constant isotropic conductivity, the surface potential at all boundaries can be found using Green's theorem (Geselowitz 1967)

$\sigma_{0} v_{\infty}(\boldsymbol{r})=\frac{\sigma_{j}^{-}+\sigma_{j}^{+}}{2} v(\boldsymbol{r})+\frac{1}{4 \pi} \sum_{i=1}^{m}\left(\sigma_{i}^{-}-\sigma_{i}^{+}\right) \int_{S_{i}} v\left(\boldsymbol{r}^{\prime}\right) \boldsymbol{n}_{i}\left(\boldsymbol{r}^{\prime}\right) \cdot \frac{\boldsymbol{r}-\boldsymbol{r}^{\prime}}{\left\|\boldsymbol{r}-\boldsymbol{r}^{\prime}\right\|^{3}} \mathrm{~d} \boldsymbol{r}^{\prime}, \quad \boldsymbol{r} \in S_{i}$

where $\boldsymbol{r}^{\prime}$ represents the source point, $\sigma_{j}^{-}$and $\sigma_{j}^{+}$represent the conductivity inside and outside of the $j$ th surface respectively, $\boldsymbol{n}_{i}\left(\boldsymbol{r}^{\prime}\right) \mathrm{d} \boldsymbol{r}^{\prime}$ is a vector element of surface $S_{i}$ oriented along the outward unit norm of $S_{i}$, and $v_{\infty}(\boldsymbol{r})$ represents the primary potential, i.e. the solution for the infinite homogeneous medium of unit conductivity $\sigma_{0}$ due to the primary current $\boldsymbol{j}^{p}(\boldsymbol{r})$ is

$$
v_{\infty}(\boldsymbol{r})=\frac{1}{4 \pi \sigma_{0}} \int_{G} \boldsymbol{j}^{p}\left(\boldsymbol{r}^{\prime}\right) \cdot \frac{\boldsymbol{r}-\boldsymbol{r}^{\prime}}{\left\|\boldsymbol{r}-\boldsymbol{r}^{\prime}\right\|^{3}} \mathrm{~d} \boldsymbol{r}^{\prime}
$$

where the integration is carried out over a closed volume $G$. Equation (1) is a Fredholm integral of the second kind for the surface potential $v(\boldsymbol{r})$. For the special case where the 
surface geometry is spherical, analytical solutions for (1) are well known (Brody et al 1973). For realistic head geometries of arbitrary shape, the surface potential $v(r)$ must be found using the computationally intensive BEM, as outlined in section 2.3 , or other numerical techniques.

Regardless of the specifics of the forward model, by electromagnetic superposition the forward model is linear in the moment, and we may write the relationship between the moment $\boldsymbol{q}$ for a dipole at $\boldsymbol{r}_{\boldsymbol{q}}$ and the measurement at sensor location $\boldsymbol{r}$ as the inner product of a 'lead field' vector $\boldsymbol{g}\left(\boldsymbol{r}, \boldsymbol{r}_{q}\right)$ and the dipole moment: $v(\boldsymbol{r})=\boldsymbol{g}\left(\boldsymbol{r}, \boldsymbol{r}_{\boldsymbol{q}}\right) \cdot \boldsymbol{q}$. We assume dipole moments of the

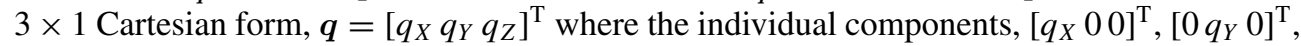
and $\left[\begin{array}{lll}0 & 0 & q_{Z}\end{array}\right]^{\mathrm{T}}$ represent 'elemental dipoles'. The three components of the lead field vector $\boldsymbol{g}\left(\boldsymbol{r}, \boldsymbol{r}_{q}\right)$ are formed as the solution to (1) for each of these elemental dipoles. Concatenating the measurements of an $m$-sensor array into a vector, we can represent the 'forward field' of the dipole as

$$
\left[\begin{array}{c}
v\left(\boldsymbol{r}_{1}\right) \\
\cdots \\
v\left(\boldsymbol{r}_{m}\right)
\end{array}\right]=\left[\begin{array}{c}
\boldsymbol{g}\left(\boldsymbol{r}_{1}, \boldsymbol{r}_{\boldsymbol{q}}\right)^{\mathrm{T}} \\
\ldots \\
\boldsymbol{g}\left(\boldsymbol{r}_{m}, \boldsymbol{r}_{\boldsymbol{q}}\right)^{\mathrm{T}}
\end{array}\right] \boldsymbol{q}=\boldsymbol{G}\left(\left\{\boldsymbol{r}_{i}\right\}, \boldsymbol{r}_{\boldsymbol{q}}\right) \boldsymbol{q}
$$

where $\boldsymbol{G}\left(\left\{\boldsymbol{r}_{i}\right\}, \boldsymbol{r}_{q}\right)$ is the 'gain matrix' relating the dipole at $\boldsymbol{r}_{\boldsymbol{q}}$ to the set of discrete sensor locations $\left\{\boldsymbol{r}_{i}\right\}$. Assuming multiple time samples, the observed set of measurements over an $m$-sensor array for $p$ dipoles can be expressed as a linear forward spatiotemporal model of the form

$$
F=G Q+N
$$

where the observed forward field $\boldsymbol{F}$ ( $m$-sensors $\times n$-time samples) can be expressed in terms of the forward model $\boldsymbol{G}$ ( $m$-sensors $\times 3 p$-elemental dipoles $)$, a set of dipole moments $Q(3 p \times n)$, and additive noise $N(m \times n)$.

When describing the rows and columns of the EEG forward gain matrix $G$, we adopt the convention described in Tripp (1983), whereby the 'lead-field' describes the flow of current for a given sensor through each of the dipole locations (and thus corresponds to each row in $G$ ), and the 'forward-field' describes the observed potential across all sensors due to an elemental dipole (and thus corresponds to each column in $G$ ).

\subsection{EEG spherical head models}

The simplest EEG head model consists of a single-layer spherical shell of uniform conductivity $\sigma$, as originally described by Wilson and Balyey (1950). A closed-form solution for calculating the potential on the outermost surface is described by Brody et al (1973). In practice, a singlelayer sphere proves too simplistic for the human head, which consists of multiple layers of conductivity varying by as much as two orders of magnitude between the skull and brain. To account for the varying conductivity of brain, skull, scalp and optionally cerebrospinal fluid, three- and four-multilayer concentric-sphere analytic solutions have been derived. These can be computed numerically using a truncated Legendre series (Cuffin and Cohen 1979). Because of their simplicity, reasonable computation requirements and relatively good accuracy, multilayer spherical models are by far the most widely used.

Methods to improve the computational efficiency of multilayer spherical models have focused primarily on approximating the infinite Legendre series. Ary et al (1981) recognized that a single-sphere model could, under certain circumstances, approximate a three-shell model with good accuracy. If we let $v^{1}\left(\boldsymbol{r} ; \boldsymbol{r}_{\boldsymbol{q}}, \boldsymbol{q}\right)$ represent a single-layer sphere and $v^{3}\left(\boldsymbol{r} ; \boldsymbol{r}_{\boldsymbol{q}}, \boldsymbol{q}\right)$ represent a three-layer sphere, then the approximation may be represented as

$$
v^{3}\left(\boldsymbol{r} ; \boldsymbol{r}_{\boldsymbol{q}}, \boldsymbol{q}\right) \cong \lambda v^{1}\left(\boldsymbol{r} ; \mu \boldsymbol{r}_{\boldsymbol{q}}, \boldsymbol{q}\right) .
$$


In other words, we can approximate $v^{3}\left(\boldsymbol{r} ; \boldsymbol{r}_{\boldsymbol{q}}, \boldsymbol{q}\right)$ by adjusting the location of the dipole along its radial direction $\boldsymbol{r}_{q} /\left|\boldsymbol{r}_{q}\right|$ by a scale factor of $\mu$, compute the much simpler single-sphere solution and then scale the solution by $\lambda$. Further refinements of this general approximation concept (Berg and Scherg 1994, deMunck and Peters 1993, Zhang 1995) resulted in the remarkably accurate approximation (Zhang 1995)

$$
v^{M}\left(\boldsymbol{r} ; \boldsymbol{r}_{q}, \boldsymbol{q}\right) \cong \sum_{j=1}^{J} \lambda_{j} v^{1}\left(\boldsymbol{r} ; \mu_{j} \boldsymbol{r}_{q}, \boldsymbol{q}\right)
$$

where $M$ is the number of shells and $J$ is the number of dipoles used (figure 2). For commonly used three- and four-shell head geometries, Berg and Scherg (1994) and Zhang (1995) found that exceptionally good approximations could be obtained using as few as $J=3$ dipoles. Zhang (1995) refers to these parameters $\mu_{j}$ and $\lambda_{j}$ as the Berg 'eccentricity' and 'magnitude' parameters respectively, and hence we will refer to this approach as the 'Berg approximation'. As for the Legendre series being approximated, the parameters $\mu_{j}$ and $\lambda_{j}$ are dependent only on the sphere radii/conductivity profile and independent of dipole position $\boldsymbol{r}_{q}$.

\subsection{Boundary element method (BEM)}

Under the assumption that the head can be modelled as a multilayer surface composed of non-intersecting homogeneous 'shells' of constant isotropic conductivity (see figure 5), the BEM can be used to solve Green's theorem (1) for the surface potential $v(r)$. The following provides a summary of the BEM approach using the method of weighted residuals, which we have reviewed for EEG and MEG applications in (Mosher et al 1999). The BEM approximates the potential function $v(r)$ as a linear combination of the $n=1, \ldots, N$ linearly independent basis functions $\varphi_{n}(\boldsymbol{r})$, a set of corresponding nodal points $\boldsymbol{r}_{n}$, and a set of corresponding unknown coefficients $\boldsymbol{v} \equiv\left[v_{1}, \ldots, v_{N}\right]^{\mathrm{T}}$ at each of the nodes, to yield

$$
v(\boldsymbol{r}) \cong \sum_{n=1}^{N} v_{n} \varphi_{n}(\boldsymbol{r}) .
$$

The most commonly used basis functions are planar triangles with either a constant potential or linearly varying potential across the surface of each triangle. The unknown coefficients therefore control these 'constant' or 'linear' approximations to the true potentials.

These approximations lead to errors in the equations that must also be controlled. Common error control methods are 'collocation' and 'Galerkin'. In collocation weighting, which is the simpler of the two methods, the error is controlled at the same discrete locations as the control points. In Galerkin weighting, the error is controlled as either a constant or linear function across the entire triangle. Using comparisons with spheres (where analytical truth can be computed), the results in Mosher et al (1999) show that the simplest linear collocation BEM is characterized by high (and somewhat erratic) error for dipoles near the inner surface boundary. In comparison, the more elaborate model provided by both the constant Galerkin and linear Galerkin forms provide significantly lower error.

In all cases, the selection of a basis and its error control lead to an $N \times N$ system of equations of the form $\boldsymbol{g}=\boldsymbol{H} \boldsymbol{v}$ whose solution for $\boldsymbol{v}$ (the unknown coefficients) can be expressed as

$$
\boldsymbol{v}=\boldsymbol{H}^{-1} \boldsymbol{g}
$$

where

$$
\boldsymbol{g}=\boldsymbol{G}_{\infty} \boldsymbol{q}=\left[\begin{array}{c}
\left(\psi_{1}(\boldsymbol{r}), \boldsymbol{k}_{\infty}\left(\boldsymbol{r}, \boldsymbol{r}_{\boldsymbol{q}}\right)\right)^{\mathrm{T}} \\
\cdots \\
\left(\psi_{N}(\boldsymbol{r}), \boldsymbol{k}_{\infty}\left(\boldsymbol{r}, \boldsymbol{r}_{\boldsymbol{q}}\right)\right)^{\mathrm{T}}
\end{array}\right] \boldsymbol{q} \quad \text { and } \quad \boldsymbol{k}_{\infty}\left(\boldsymbol{r}, \boldsymbol{r}_{\boldsymbol{q}}\right)=\left[\frac{1}{4 \pi} \frac{\boldsymbol{r}-\boldsymbol{r}_{\boldsymbol{q}}}{\left|\boldsymbol{r}-\boldsymbol{r}_{\boldsymbol{q}}\right|^{3}}\right]
$$


$\psi_{n}(r)$ represents the weighting basis function, and the operator $(f(r), g(r))$ denotes the inner product of the functions $f$ and $g$ formed by integrating over the surfaces. The potential $v(\boldsymbol{r})$ function for a surface point can then be found from (7) as a linear combination of these coefficients:

$$
v(\boldsymbol{r}) \cong\left[\varphi_{1}(\boldsymbol{r}), \ldots, \varphi_{N}(\boldsymbol{r})\right] \boldsymbol{v}=\left(\left[\varphi_{1}(\boldsymbol{r}), \ldots, \varphi_{N}(\boldsymbol{r})\right] \boldsymbol{H}^{-1}\right)\left(\boldsymbol{G}_{\infty} \boldsymbol{q}\right) .
$$

As shown in Mosher et al (1999) and implied in (10), we can further partition the solution into 'subject-dependent' and 'dipole-dependent' terms. Computational savings can be realized by performing a once-per-subject computation of the subject-dependent terms $\left(\left[\varphi_{1}(\boldsymbol{r}), \ldots, \varphi_{N}(\boldsymbol{r})\right] \boldsymbol{H}^{-1}\right)$ (commonly referred to as the BEM 'transfer matrix') and then at runtime computing only the dipole-dependent terms in the matrix $\boldsymbol{G}_{\infty}$. While these computational savings are significant, the computational requirement for the BEM is still significantly higher than that of a multilayer sphere. Consequently, use of the BEM for the inverse problem is typically viewed as computationally prohibitive, particularly in nonlinear directed-search algorithms which must repeatedly evaluate thousands of putative source solutions.

\section{Methods}

As an alternative to the computationally intensive BEM forward model calculations described in equations (8)-(10), we propose the following two methods. Each method provides an approximation to the BEM which is significantly faster to compute at run-time. One-time precalculation of the BEM solution over a limited set of dipole locations is required for each method. In our experiments, we use the more accurate constant Galerkin and linear Galerkin BEM forms. In particular, we used the computationally intensive linear Galerkin form in order to establish our best estimate of truth for realistic head geometries. We also performed computations using the isolated skull approach (ISA) described by Hämäläinen and Sarvas (1989).

Our first method extends the MEG sensor-fitted sphere approach presented by Huang et al (1999) to the EEG forward model. The objective in Huang et al (1999) is to find the 'optimally fit' sphere at each sensor that best approximates the true lead-field for the actual head volume. In our approach, we estimate the true lead-field by computing the BEM over a representative dipole grid. Sensor-fitted sphere parameters are then determined using the methodology described in section 3.1. Our second method consists of a straightforward 3D forward-field interpolation scheme, again computing the BEM over a representative dipole grid. This method is described in section 3.2.

\subsection{Sensor fitted sphere}

A schematic diagram of the sensor-fitted sphere model described in Huang et al (1999) is shown in figure 1 . The goal of this method is to determine the centre $C_{0}$ and outer radius $R_{0}$ of the fitted-sphere that best approximates the true lead field for each individual sensor. We first compute the lead field for a given sensor located at $\boldsymbol{r}$ over a representative grid of $P$ dipoles $\boldsymbol{r}^{\prime}=\left\{\boldsymbol{r}_{1}^{\prime}, \boldsymbol{r}_{2}^{\prime}, \ldots, \boldsymbol{r}_{P}^{\prime}\right\}$ using a high-fidelity EEG forward model solution. In our case, we used the linear Galerkin BEM form described in Mosher et al (1999), using layer conductivities of $\underline{\sigma}=\left\{\sigma_{1}, \ldots, \sigma_{M}\right\}$. Assuming a multilayer sphere characterized by conductivity values $\underline{\sigma}$ and sphere radii $R_{0} \underline{\rho}=\left\{\rho_{1} R_{0}, \ldots, \rho_{M} R_{0}\right\}$ with fixed relative radii $\rho_{1}, \ldots \rho_{M}$, we find the multilayer sphere centre $C_{0}$ and outer radius $R_{0}$ for each sensor as

$$
\left\{R_{0}, C_{0}\right\}=\operatorname{argmin}\left(\left\|g_{\mathrm{sph}}\left(\boldsymbol{r}, \boldsymbol{r}^{\prime}, \underline{\sigma}, \underline{\rho} R_{0}\right)-\boldsymbol{g}_{\mathrm{BEM}}\left(\boldsymbol{r}, \boldsymbol{r}^{\prime}, \underline{\sigma}\right)\right\|^{2}\right)
$$


where $\boldsymbol{g}_{\mathrm{sph}}\left(\boldsymbol{r}, \boldsymbol{r}^{\prime}, \underline{\sigma}, \underline{\rho} R_{0}\right)$ and $\boldsymbol{g}_{\mathrm{BEM}}\left(\boldsymbol{r}, \boldsymbol{r}^{\prime}, \underline{\sigma}\right)$ are the lead field vectors for the spherical and BEM solutions respectively. Since previously described multilayer spherical models require the sensor to lie on the outermost surface, we impose the constraint that $\left|\boldsymbol{r}-C_{0}\right|=R_{0}$. Solution of the sphere parameters using (11) is repeated for each of the $m$ sensors. The EEG sensor-fitted sphere forward model is then expressed as

$$
\boldsymbol{G}_{\mathrm{OS}}=\left[\begin{array}{c}
\boldsymbol{g}_{\mathrm{sph}}\left(\boldsymbol{r}_{1}, \boldsymbol{r}^{\prime}, \underline{\sigma}, \underline{\rho} R_{0}(1)\right) \\
\ldots \\
\boldsymbol{g}_{\mathrm{sph}}\left(\boldsymbol{r}_{M}, \boldsymbol{r}^{\prime}, \underline{\sigma}, \underline{\rho} R_{0}(m)\right)
\end{array}\right] .
$$

The use of a proportional radii model allows for efficient implementation of the Berg approximation. The fitting of sphere parameters using (11) requires a significant number of lead-field evaluations using a multilayer sphere model. As noted in section 2.2, the Berg approximation is a computationally efficient alternative to the truncated Legendre expansion. Under the assumption that all spheres have the same fixed isotropic conductivities and fixed proportional radii $\rho=\left\{\rho_{1}, \ldots, \rho_{M}\right\}$, calculation of the Berg parameters $\mu_{j}$ and $\lambda_{j}$ need only be performed once.

3.1.1. Approximation of potentials for sources external to a fitted sphere. Approximation of the head volume with multiple spheres also presents the problem of incomplete head coverage. As illustrated in figure 1, an anatomically fit sphere for an occipital sensor does not cover the frontal regions. Thus a frontal neural source is undefined for the occipital sensor, since the source is external to the model sphere. In general, for overlapping multiple spheres fitted to each sensor, we will find regions of the brain that are undefined for some subset of the sensor array. Typically, these 'external' sources are quite distant from the sensors for which they are undefined, and one may argue that the small contributions of these sources to the distant sensor could simply be set to zero. The problem lies in the resulting discontinuous jump to a zero potential for dipoles just inside and outside all of these local spheres, which may cause difficulties in source localization procedures using a nonlinear search algorithm. In practice, we would like to have a model defined at all spatial locations that is compatible with nonlinear directed search algorithms, so that for a dipole traversing any sphere boundary, the potential described by the forward model is continuous. The directed searches are allowed to continue across sphere boundaries to find a solution in a physically plausible location.

To achieve this continuity in potential, we first use the previously described Berg approximation to reduce the EEG forward model complexity from a multilayer sphere to a linear combination of simpler (closed-form) single-sphere models, as illustrated in figure 2. We then apply the method of images (Brody et al 1973, Cheng 1989) to generate a plausible solution that allows for a continuous approximation across the single-shell boundary. We apply the method of images analogous to the electromagnetic problem of a point charge in the presence of a spherical conductor. For our purposes, we represent a dipole external to a fitted sphere by a 'similar' dipole that falls within the boundaries of the sphere. Referring to figure 3, we consider a point current source of strength $q_{0}$ located a distance $r_{0}$ from the centre of a homogeneous spherical conducting volume of radius $R$ and conductivity $\sigma$. An image source of strength $q_{i}=R q_{0} / r_{0}$ is located on the radius vector of the point source at a distance $r_{i}=R^{2} / r_{0}$ (Cheng 1989).

For dipoles interior to the sphere $r_{q} \leqslant R$, we use the standard single-shell forward model to compute $v^{1}\left(\boldsymbol{r} ; \boldsymbol{r}_{q}, \boldsymbol{q}_{0}\right)$. For those dipoles external to the sphere $r_{q}>R$, we can approximate the forward model in terms of the source image as

$$
v^{1}\left(\boldsymbol{r} ; \boldsymbol{r}_{q}, \boldsymbol{q}_{0}\right) \approx v^{1}\left(\boldsymbol{r} ; \frac{R^{2}}{r_{q}^{2}} \boldsymbol{r}_{q}, \frac{R}{r_{q}} \boldsymbol{q}_{0}\right) \text {. }
$$




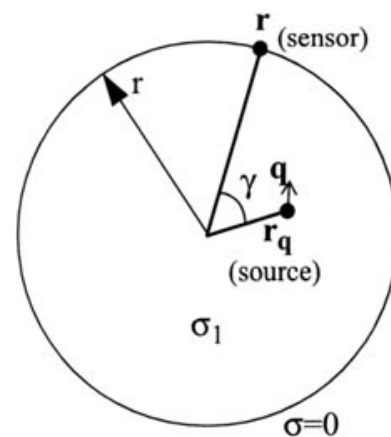

A) Single Layer Sphere

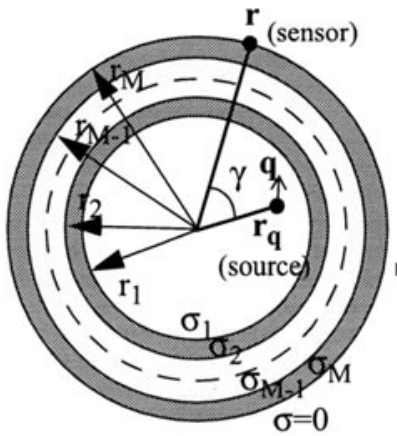

B) Multi-Layer Concentric Sphere

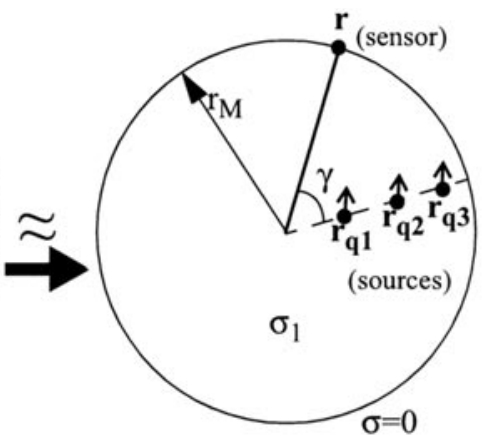

C) Multi-Layer Concentric Sphere Approximation Using Berg Model

Figure 2. Graphical depiction of concentric sphere models and parameter definitions.
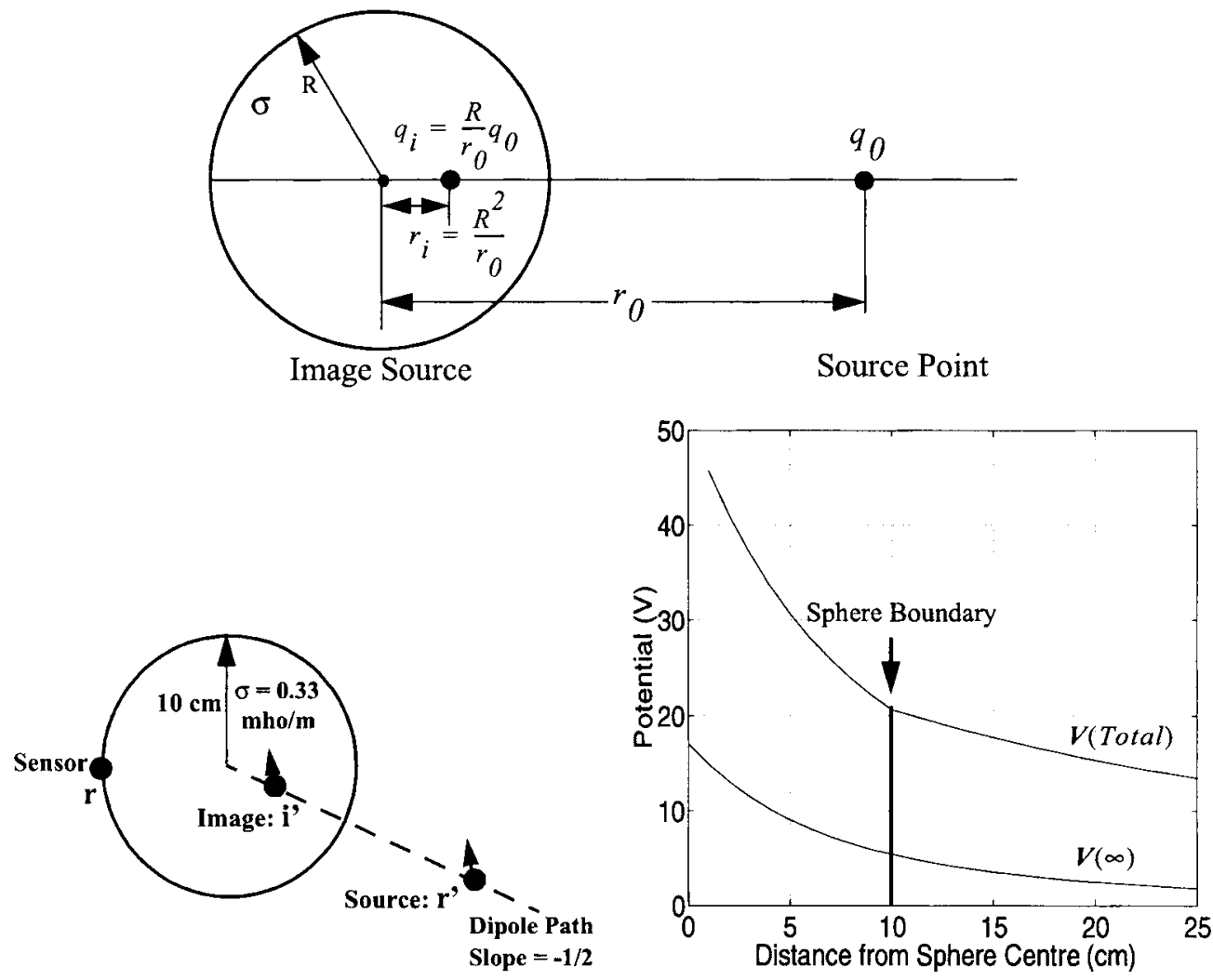

Figure 3. (Upper) Calculation of electric 'image' of an external current dipole. (Lower left) Representative example where dipole traverses across sphere boundary. Electrical image is used to represent dipoles external to the sphere. (Lower right) Computed potential versus dipole position for representative example.

A representative example is shown in figure 3 using a dipole that traverses across a sphere boundary. The resulting approximation, although fictitious, is plausible and continuous across the sphere boundary, and the forward model is now defined at all spatial locations. 

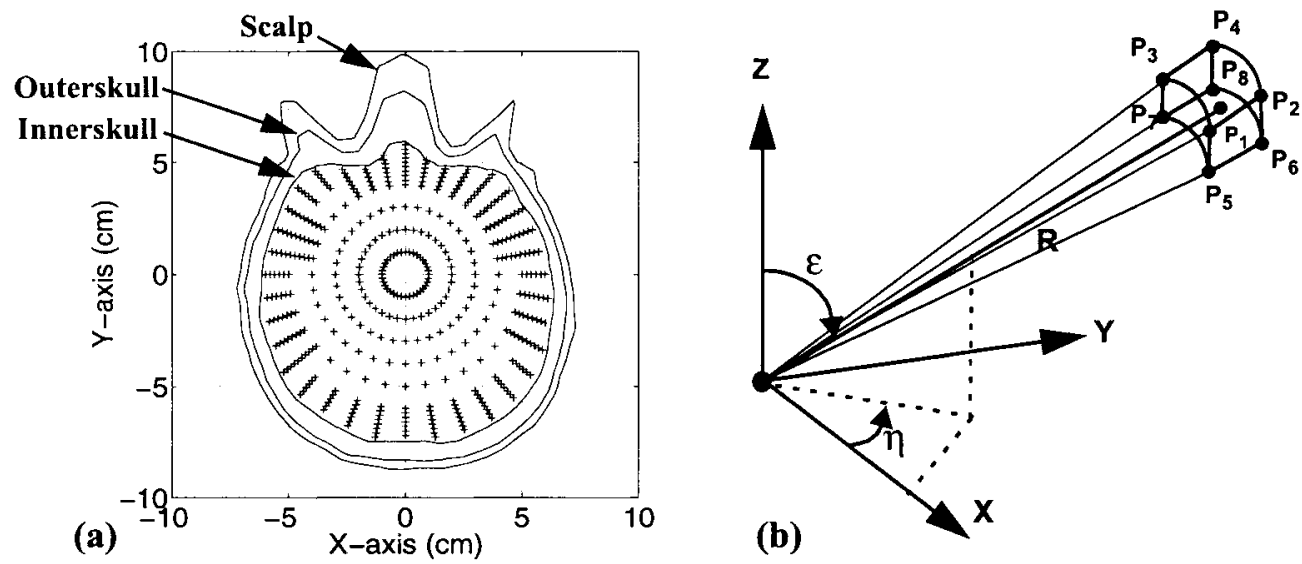

Figure 4. (a) Spherical grid points denoted as + at level $Z=0$. (b) Forward-field interpolation at point $(\eta, \varepsilon, R)$ based on weighted sum of eight nearest grid points.

\subsection{Three-dimensional forward-field interpolation}

The three-dimensional forward-field interpolation method is illustrated in figure 4 . We first define a set of grid points sampled in spherical coordinates $(\eta, \varepsilon, R)$ throughout the inner skull cavity. In our study we sampled both azimuth $\eta$ and elevation $\varepsilon$ at $10^{\circ}$ intervals. This corresponded to a maximum cross-range of $1.4 \mathrm{~cm}$ at a radius of $8 \mathrm{~cm}$. Sampling in the radial dimension was performed using a two-tiered scheme. The skull boundary for each azimuthelevation ray was readily determined by finding the intersection point on the surface triangle penetrated by the ray. Within 1-2 cm of the inner skull boundary we sampled at $2 \mathrm{~mm}$ intervals, and at $1 \mathrm{~cm}$ intervals elsewhere. We used a finer radial sampling in the vicinity of the surface boundary to account for the increased sensitivity of the forward field to variations in source locations within this region.

During the precomputation phase we use a BEM method to compute the forward field at each grid position. We then store each result as an indexed table for use by the interpolator during the inverse procedure. At run-time, we determine the EEG forward model at an arbitrary location using a three-dimensional trilinear interpolation of the forward-field solution corresponding to the eight nearest grid points

$$
v\left(\left\{\boldsymbol{r}_{i}\right\}, \boldsymbol{r}_{\boldsymbol{q}}, \boldsymbol{q}\right) \cong\left[\sum_{i=1}^{8} w_{i} \boldsymbol{G}\left(\left\{\boldsymbol{r}_{i}\right\}, \boldsymbol{r}_{\boldsymbol{q} i}\right)\right] \boldsymbol{q}
$$

where, from (3), $\boldsymbol{G}\left(\left\{\boldsymbol{r}_{i}\right\}, \boldsymbol{r}_{q i}\right)$ represents the precomputed $m \times 3$ forward-field gain matrix at the $i$ th grid point $\boldsymbol{r}_{q i}$, and $\left\{\boldsymbol{r}_{i}\right\}$ is the set of $m$ sensor positions. For a dipole with spherical coordinates $\boldsymbol{r}_{q}=(\eta, \varepsilon, R)$, the trilinear interpolation weights $w_{i}$ corresponding to each of the eight grid points $\boldsymbol{r}_{q i}=\left(\eta_{i}, \varepsilon_{i}, R_{i}\right)$ shown in figure 4 are computed as

$$
\begin{aligned}
& w_{1}=\left[1-\left(\frac{\varepsilon-\varepsilon_{1}}{\varepsilon_{5}-\varepsilon_{1}}\right)\right]\left[1-\left(\frac{\eta-\eta_{1}}{\eta_{3}-\eta_{1}}\right)\right]\left[1-\left(\frac{R-R_{1}}{R_{2}-R_{1}}\right)\right] \\
& w_{2}=\left[1-\left(\frac{\varepsilon-\varepsilon_{2}}{\varepsilon_{6}-\varepsilon_{2}}\right)\right]\left[1-\left(\frac{\eta-\eta_{2}}{\eta_{4}-\eta_{2}}\right)\right]\left(\frac{R-R_{1}}{R_{2}-R_{1}}\right)
\end{aligned}
$$




$$
\begin{aligned}
& w_{3}= {\left[1-\left(\frac{\varepsilon-\varepsilon_{3}}{\varepsilon_{7}-\varepsilon_{3}}\right)\right]\left(\frac{\eta-\eta_{1}}{\eta_{3}-\eta_{1}}\right)\left[1-\left(\frac{R-R_{3}}{R_{4}-R_{3}}\right)\right] } \\
& w_{4}= {\left[1-\left(\frac{\varepsilon-\varepsilon_{4}}{\varepsilon_{8}-\varepsilon_{4}}\right)\right]\left(\frac{\eta-\eta_{2}}{\eta_{4}-\eta_{2}}\right)\left(\frac{R-R_{3}}{R_{4}-R_{3}}\right) } \\
& w_{5}=\left(\frac{\varepsilon-\varepsilon_{1}}{\varepsilon_{5}-\varepsilon_{1}}\right)\left[1-\left(\frac{\eta-\eta_{5}}{\eta_{7}-\eta_{5}}\right)\right]\left[1-\left(\frac{R-R_{5}}{R_{6}-R_{5}}\right)\right] \\
& w_{6}=\left(\frac{\varepsilon-\varepsilon_{2}}{\varepsilon_{6}-\varepsilon_{2}}\right)\left[1-\left(\frac{\eta-\eta_{6}}{\eta_{8}-\eta_{6}}\right)\right]\left(\frac{R-R_{5}}{R_{6}-R_{5}}\right) \\
& w_{7}=\left(\frac{\varepsilon-\varepsilon_{3}}{\varepsilon_{7}-\varepsilon_{3}}\right)\left(\frac{\eta-\eta_{5}}{\eta_{7}-\eta_{5}}\right)\left[1-\left(\frac{R-R_{7}}{R_{8}-R_{7}}\right)\right] \\
& w_{8}=\left(\frac{\varepsilon-\varepsilon_{4}}{\varepsilon_{8}-\varepsilon_{4}}\right)\left(\frac{\eta-\eta_{6}}{\eta_{8}-\eta_{6}}\right)\left(\frac{R-R_{7}}{R_{8}-R_{7}}\right) .
\end{aligned}
$$

Although higher-order interpolation methods can potentially improve accuracy, we use a simple trilinear method. The higher-order schemes involve greater computation cost and the results presented below indicate that they are probably not necessary with the sampling density that we are using.

The quality of the post-interpolated solution is highly dependent on the quality of the original forward model used to compute the grid. As shown in (Mosher et al 1999), the quality of various BEM solutions is highly dependent on the weighting function used. While faster to compute, simpler models like linear collocation BEM show increased error and a somewhat erratic solution near the innermost surface boundary (cortical region). Consequently, interpolated results were also poor. For our experiments, we used the more accurate constant and linear Galerkin BEM forms.

The grid sampling needs to be sufficiently dense in rapidly changing regions and must cover all regions of interest in the brain. To ensure that all dipole interpolations fall within our precomputed grid, we sampled the radial dimension to within $2 \mathrm{~mm}$ of the inner skull boundary. In choosing grid sampling resolution, a trade-off exists between interpolation accuracy and the required grid size. As noted earlier, we implemented a scheme using a denser radial sampling in cortical regions where the potential function was particularly sensitive. We found that the angular dimensions were far less sensitive and opted for an angular resolution of $10^{\circ}$. Tests in sections 4.1 and 4.2 were also performed using a finer grid angular resolution of $5^{\circ}$. While computation and memory requirements grew by a factor of four, the improvement in performance was negligible.

Finally, the key to achieving fast recomputation times was an efficient grid indexing scheme. For a dipole located at $(\eta, \varepsilon, R)$, our gridding scheme was designed so that the indices of the eight nearest neighbours could be determined at run-time using trivial modulo operations, averting the need for complex searches and distance calculations to find the nearest neighbours.

\section{Results}

We used the following metrics to evaluate pair-wise forward-field scale and subspace errors between the true forward gain matrix $\boldsymbol{G}_{A}$ and our approximation $\boldsymbol{G}_{B}$. We denote the $m$-sensor forward field gain vector for the $n$th elemental dipole as $g_{A}(n)$ for $n=1, \ldots, 3 p$ elemental dipoles.

(a) Pairwise forward field PVU $(0 \leqslant$ PVU $\leqslant 100 \%)$ : the purpose of this metric ('percent variance unexplained') is to evaluate scale error and serve as a predictor of forward model 
performance using least-squares based approaches

$$
\operatorname{PVU}(n)=\frac{\left\|\boldsymbol{g}_{B}(n)-\boldsymbol{g}_{A}(n)\right\|^{2}}{\left\|\boldsymbol{g}_{A}(n)\right\|^{2}} \times 100 \% \quad n=1, \ldots, 3 p .
$$

(b) Pairwise forward field subspace correlation: the purpose of this metric is to evaluate subspace error which serves as a predictor of forward model performance when using subspace-based approaches like MUSIC (Mosher et al 1992) and RAP-MUSIC (Mosher and Leahy 1999)

$\operatorname{SBC}_{\mathrm{FF}}(n)=\operatorname{subcorr}\left(\boldsymbol{g}_{A}(n), \boldsymbol{g}_{B}(n)\right)=\frac{\boldsymbol{g}_{A}^{\mathrm{T}} \boldsymbol{g}_{B}}{\left\|\boldsymbol{g}_{A}\right\|\left\|\boldsymbol{g}_{B}\right\|} \quad n=1, \ldots, 3 p$

where $\operatorname{subcorr}(\boldsymbol{a}, \boldsymbol{b})$ represents the cosine of the angle between the vectors $\boldsymbol{a}$ and $\boldsymbol{b}$. Whereas the PVU metric is dependent on both magnitude and orientation differences between the two vectors, the subcorr metric is strictly dependent on differences in orientation between the two vectors.

We avoided specifying performance in terms of localization accuracy, which is subject to the influence of arbitrary design parameters (e.g. error thresholds, localization methodology, etc). Instead, metrics (19) and (20) directly compare the forward model solution with our best estimate of truth. These metrics, PUV and subcorr, reflect the base criteria used to localize sources using, respectively, least-squares and subspace based localization methods, and thus have a direct bearing on localization accuracy.

\subsection{Spherical model comparisons}

To evaluate the impact of errors directly attributable to trilinear interpolation, we conducted a simple experiment using a three-layer sphere of increasing radius $8.1,8.5$ and $8.8 \mathrm{~cm}$ with conductivities $0.33,0.0042$ and $0.33 \Omega^{-1}$ respectively. A three-dimensional interpolative grid was set up using the sampling scheme described in section 3.2. The potential at each grid point was evaluated using a multilayer sphere model whose true forward solution was determined analytically.

We then generated a set of test dipoles falling at the centre of each spherical voxel as illustrated in figure $4(b)$. Dipoles within $3 \mathrm{~mm}$ of the inner layer boundary were included to simulate gyral cortical sources. The true forward model for each test dipole was again computed using an analytical multilayer sphere model. We then evaluated the interpolated solution performance using the PVU and subcorr metrics described in section 4.

While not shown here, the pairwise scale and subspace errors were extremely small (PVU $<1 \%$ and subcorr $>0.99$ ) for all elemental dipoles. Our conclusion is that the potential function is sufficiently flat that trilinear interpolation of the forward field over a reasonably dense grid imposes little distortion with respect to the true solution.

\subsection{Phantom experiments}

We used surface data from the human skull phantom described in Leahy et al (1998) to evaluate algorithm performance over a realistic head shape (figure 5). Using X-ray CT data, three surfaces (inner skull, outer skull, scalp) were tessellated to a density of 1016 triangles per layer. Measured brain, skull, and scalp conductivities of $0.40,0.004$ and $0.21 \Omega^{-1}$ respectively were assumed to be uniform and isotropic. A representative set of 1622 randomly located test dipoles (4866 elemental dipoles) were generated, where all dipoles were internal to both the inner skull and 'best-fitted sphere' volumes. To evaluate model performance in gyral regions, 


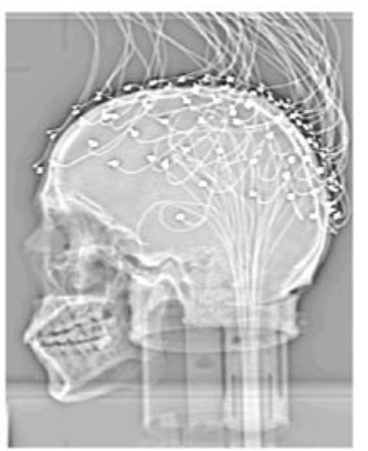

A) X-ray CT view of USC/LANL human skull phantom

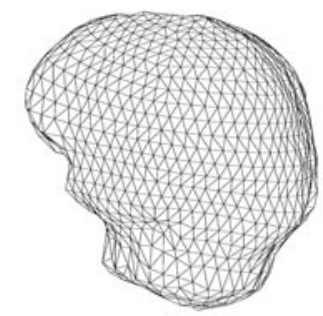

Surface layer extraction of brain cavity (at inner skull surface) via X-ray CT

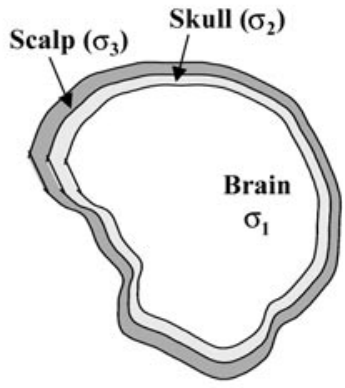

C) 3-Layer constant conductivity model over contiguous isotropic regions

Figure 5. Physical model used in BEM modelling of human skull phantom.

we allowed dipole positions to fall within $3 \mathrm{~mm}$ of the inner skull boundary. The sensor array consisted of 54 sensors distributed uniformly over the upper portion of the phantom scalp. To establish a best estimate of EEG forward model 'truth', we computed the linear Galerkin BEM solution for each test dipole. Comparisons of the linear Galerkin BEM solution using tessellated multilayer spheres (where analytical 'truth' can be determined) show typical PVU error of $1 \%$ and worst case PVU error of 6\% (Mosher et al 1999). With performance significantly better than other BEM forms; we therefore use the linear Galerkin BEM solution as our 'gold standard' for the true potential over an arbitrarily shaped head.

Scale and subspace error metrics for each elemental dipole are shown in figure 6 for the different forward models described above. To evaluate the dependence of error in the EEG forward model on spatial location, scale and subspace error were also evaluated over a fixed surface lying $1 \mathrm{~cm}$ below the innerskull surface (figure 7). To represent error over three dimensions, scale and subspace metrics in (19) and (20) were modified as follows. For scale errors, the PVU metric in (19) was computed for the ( $m$-sensors $\times 3$-elemental dipole) forward gain matrix $\boldsymbol{G}\left(\{\boldsymbol{r}\}, \boldsymbol{r}_{q k}\right) ; k=1, \ldots, p$ at each of the $p$ dipole locations as

$\operatorname{PVU}(k)=\frac{\left\|\boldsymbol{G}_{B}\left(\{\boldsymbol{r}\}, \boldsymbol{r}_{\boldsymbol{q} k}\right)-\boldsymbol{G}_{A}\left(\{\boldsymbol{r}\}, \boldsymbol{r}_{\boldsymbol{q} k}\right)\right\|_{\mathrm{Fro}}^{2}}{\left\|\boldsymbol{G}_{A}\left(\{\boldsymbol{r}\}, \boldsymbol{r}_{\boldsymbol{q}}\right)\right\|_{\mathrm{Fro}}^{2}} \times 100 \% \quad k=1, \ldots, p$

where $\|\boldsymbol{G}\|_{\text {Fro }}$ represents the Frobenius matrix norm.

For subspace errors, we again compare the forward gain matrix $\boldsymbol{G}\left(\{\boldsymbol{r}\}, \boldsymbol{r}_{q k}\right)$ with our best estimate of truth, where subcorr $\left(\boldsymbol{G}_{A}\left(\{\boldsymbol{r}\}, \boldsymbol{r}_{q k}\right), \boldsymbol{G}_{B}\left(\{\boldsymbol{r}\}, \boldsymbol{r}_{q k}\right)\right)_{\min }$ represents the cosine of the largest principal angle between the two $(m \times 3)$ subspaces (Mosher and Leahy 1999). This metric corresponds to worst possible subspace error over all possible source orientations at any given spatial location.

\subsection{Computational and memory comparisons}

Timing metrics for various EEG forward modelling methods are shown in table 1. All timing benchmarks were determined running double-precision MATLAB-based programs (The MathWorks, Natick, MA) on a $500 \mathrm{MHz}$ Pentium P-III PC with $512 \mathrm{MB}$ of RAM. These metrics correspond to the results presented in figure 6 for the phantom experiment described in section 4.2. The results show a three-dimensional forward model recomputation 


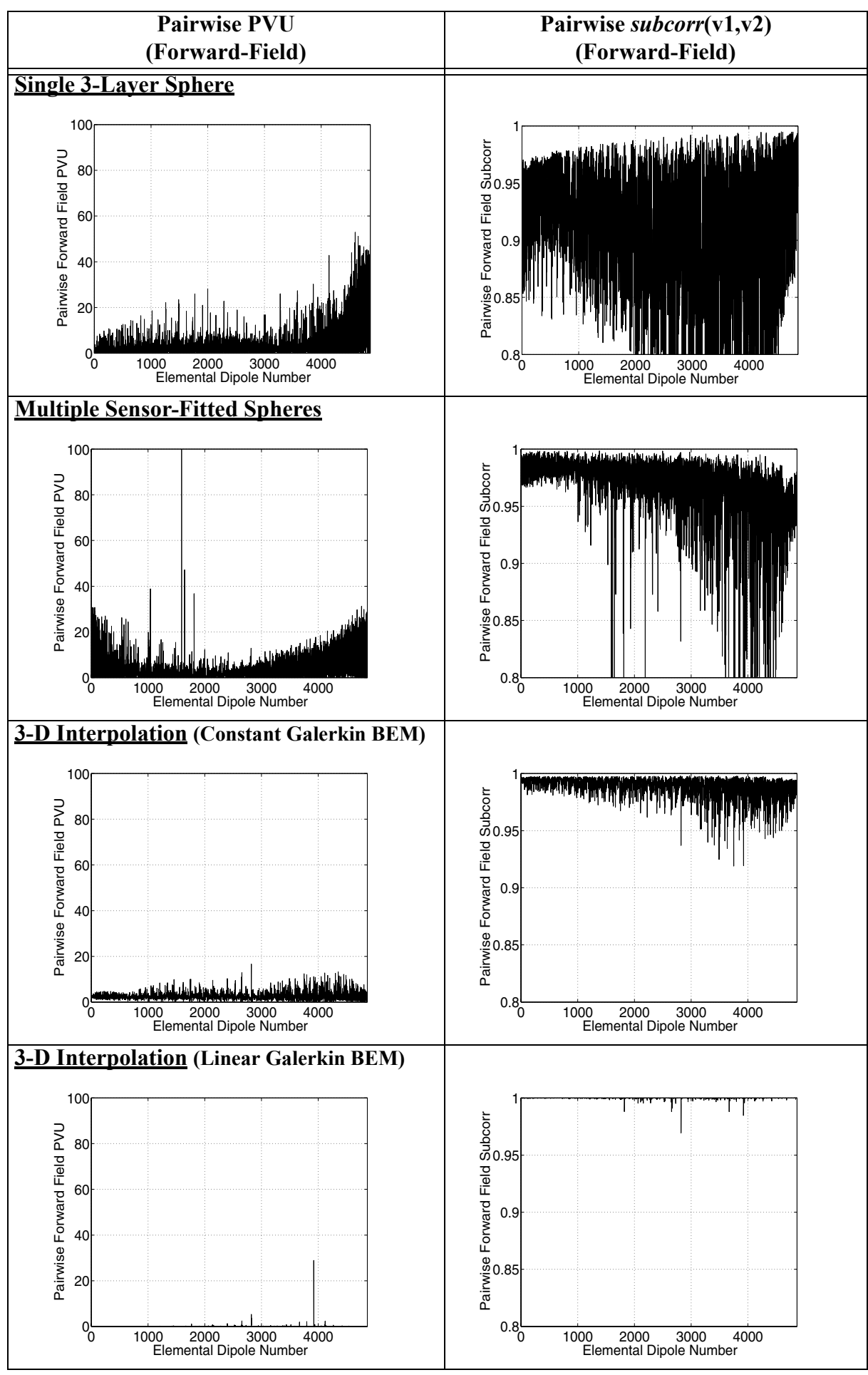

Figure 6. Pairwise forward-field PVU (left) and pairwise forward-field subspace correlation (right) between candidate EEG forward model and linear Galerkin BEM. 


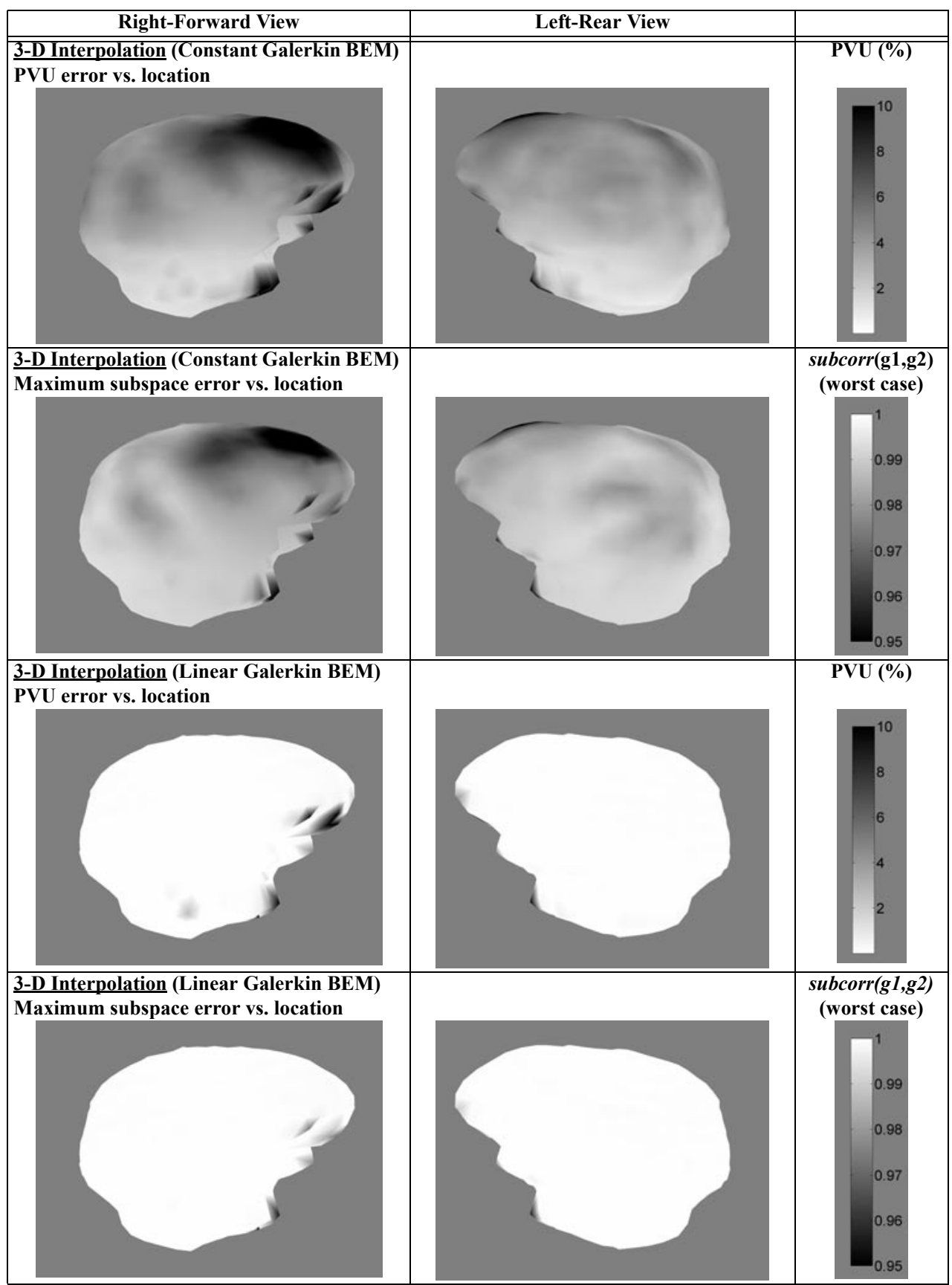

Figure 7. Spatial representation of forward-field PVU and worst-case forward-field subspace errors at fixed surface depth of $1 \mathrm{~cm}$ below inner skull for 3D interpolation cases. Error is minimal with the exception of the eye socket region and frontal head area near the sensors.

time that is in excess of 30 times faster than that of a traditional multilayer spherical model. One-time calculations required for each subject were also reasonable. 
Table 1. Computation and memory benchmarks for various EEG forward models using USC/LANL human skull phantom ${ }^{\mathrm{a}}$ and 1622 dipole test set.

\begin{tabular}{lccc}
\hline & $\begin{array}{l}\text { One-time } \\
\text { precalculations } \\
\text { (s) }\end{array}$ & $\begin{array}{l}\text { Memory } \\
\text { storage } \\
(\mathrm{MB})\end{array}$ & $\begin{array}{l}\text { 1622 dipole } \\
\text { evaluation } \\
\text { time (s) }\end{array}$ \\
\hline $\begin{array}{l}\text { BEM forward model method } \\
\text { (linear collocation) }\end{array}$ & 382.8 & 2.6 & 55.5 \\
$\begin{array}{l}\text { BEM } \\
\text { (constant Galerkin) }\end{array}$ & 3141.5 & 2.6 & 490.9 \\
$\begin{array}{l}\text { BEM } \\
\text { (linear Galerkin) }\end{array}$ & 24778.3 & 2.6 & 1667.6 \\
$\begin{array}{l}\text { Three-layer sphere } \\
\text { (100-term Legendre expansion) }\end{array}$ & 0.0 & 0.0 & 41.2 \\
$\begin{array}{l}\text { Three-layer sphere } \\
\text { (three-dipole Berg approx.) }\end{array}$ & 34.2 & 0.0 & 2.36 \\
$\begin{array}{l}\text { Multiple sensor-fitted } \\
\text { spheres }\end{array}$ & 23035.7 & 0.0 & 5.97 \\
$\begin{array}{l}\text { 3D interpolation } \\
\text { (constant Galerkin BEM) }\end{array}$ & 5748.1 & 11.7 & 1.34 \\
$\begin{array}{l}\text { 3D interpolation } \\
\text { (linear Galerkin BEM) }\end{array}$ & 33712.6 & 11.7 & 1.34 \\
\hline
\end{tabular}

a USC/LANL Neuroimaging Website: http://neuroimage.usc.edu (University of Southern California Signal and Image Processing Institute and Los Alamos National Laboratories).

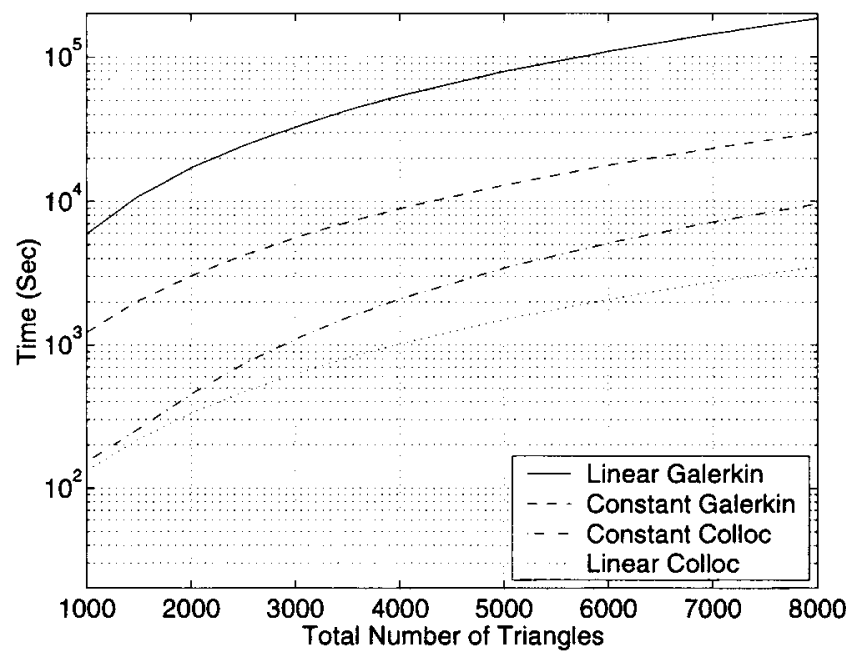

Figure 8. Computational requirements for computing the EEG BEM transfer matrix and the EEG BEM kernel evaluation at 8475 interpolative grid points for the four BEM methods described in Mosher et al (1999). The above represents total one-time computations required for the human skull phantom using a 54 sensor array. Timing benchmarks were determined using a $500 \mathrm{MHz}$ Pentium P-III PC with 512 MB RAM running double-precision MATLAB based programs.

Total grid precomputation time using each of the BEM methods in (Mosher et al 1999) is shown in figure 8 as a function of the total number of tesselation elements. This metric reflects total one-time precomputation required, for each subject and sensor geometry, to determine BEM transfer matrices and evaluate the kernel for 8475 grid points. 


\section{Discussion}

Both of the three-dimensional spherical interpolation models shown in figure 6 significantly outperform the traditional single multilayer sphere model in terms of subspace error and PVU metrics. Constant and linear Galerkin BEM forms were observed to have subspace correlations better than 0.95 in nearly all cases. In comparison, the subspace correlations using a single best-fitted multilayer sphere often fell below 0.9. A reduction in PVU scale error was also observed. A somewhat less dramatic EEG forward modelling improvement was obtained using the multiple fitted-sphere approach in section 3.1. Realistic EEG forward models have the added advantage of being defined over the whole head. In comparison, the surface potential for a dipole located external to a locally fitted sphere, yet still inside the skull, is undefined in a fitted sphere model, and requires some form of approximation as we have presented here.

Using a multilayer sphere and realistic skull phantom-based geometry, we observed that three-dimensional interpolation of the EEG forward field over a reasonably sampled grid produced very accurate approximations to the true forward field. This was the case even within $3 \mathrm{~mm}$ of the boundary of the inner skull surface. Exceptions were found only near rapidly changing surface boundaries (e.g. the eye socket region) where the numerical solutions vary rapidly. In the case of less accurate BEM methods (e.g. the constant Galerkin BEM), we also observed a slight increase in both PVU and subspace error in the superior-frontal regions of the head.

The spherical three-dimensional forward-field interpolation presented is more than 30 times faster to compute than the traditional multilayer spherical forward model. We note that the forward model recomputation time using the three-dimensional interpolation model is independent of the specific BEM method chosen. While our presentation emphasized BEM methods, the method can be generalized to other EEG forward models. Cast in this framework, high-fidelity numerical solutions currently viewed as computationally prohibitive for solving the inverse problem (e.g. our 'gold standard' linear Galerkin BEM (Mosher et al 1999)) can be rapidly recomputed in a highly efficient manner. One-time computational costs associated with determining the BEM solution over a pre-defined grid are not prohibitive (figure 8), falling within the 1 to $10 \mathrm{~h}$ range depending on the BEM method chosen and the total number of triangles used to represent all subject surfaces.

Although the three-dimensional interpolation method presented above focuses on the EEG forward problem, the method is directly applicable to the MEG analysis as well. While not presented here, our tests demonstrated MEG performance similar to the EEG results presented in section 4. An exception was for sources near the centre of the head, where the MEG fields diminish rapidly, resulting in increased interpolation error in our coarser grids. While this area is typically of limited interest, these sensitivities can be countered using finer radial grid sampling in the vicinity of this region.

\section{References}

Ary J P, Klein S A and Fender D H 1981 Location of sources of evoked scalp potentials: corrections for skull and scalp thickness IEEE Trans. Biomed. Eng. 28 447-52

Baillet S, Mosher J C and Leahy R M 2000 BrainStorm beta release: a Matlab software package for MEG signal processing and source localization and visualization Neuroimage 11 S915

Berg P and Scherg M 1994 A fast method for forward computation of multiple-shell spherical head models Electroencephalogr Clin. Neurophysiol. 90 58-64

Brody D A, Terry F H and Ideker R E 1973 Eccentric dipole in a spherical medium: generalized expression for surface potentials IEEE Trans. Biomed. Eng. 20 141-3

Cheng D K 1989 Field and Wave Electromagnetics 2nd edn (Reading, MA: Addison-Wesley) pp 141-3 
Cuffin B N and Cohen D 1979 Comparison of the magnetoencephalogram and electroencephalogram Electroencephalogr. Clin. Neurophysiol. 47 132-46

deMunck J C and Peters M J 1993 A fast method to compute the potential in the multisphere model IEEE Trans. Biomed. Eng. 40 1166-74

Ermer J J, Mosher J C, Baillet S and Leahy R M 2000 Rapidly re-computable EEG forward models for realistic head shapes Biomag 2000: Proc. 12th Int. Conf. on Biomagnetism (Espoo, Finland, 13-17 August 2000) at press

Geselowitz D B 1967 Green's theorem and potentials in a volume conductor IEEE Trans. Biomed. Eng. 14 54-5

Hämäläinen M and Sarvas J 1989 Realistic conductor geometry model of the human head for interpretation of neuromagnetic data IEEE Trans. Biomed. Eng. 36 165-71

Huang M X, Mosher J C and Leahy R M 1999 A sensor-weighted overlapping-sphere head model and exhaustive head model comparison for MEG Phys. Med. Biol. 44 423-40

Leahy R M, Mosher J C, Spencer M E, Huang M X and Lewine J D 1998 A study of dipole localization accuracy for MEG and EEG using a human skull phantom Electroencephalogr. Clin. Neurophysiol. 107 159-73

Mosher J C and Leahy R M 1999 Source localization using recursively applied and projected (RAP) MUSIC IEEE Trans. Signal Process. 47 332-40

Mosher J C, Leahy R M and Lewis P S 1999 EEG and MEG: forward solutions for inverse methods IEEE Trans. Biomed. Eng. 46 245-59

Mosher J C, Lewis P S and Leahy R M 1992 Multiple dipole modelling and localization from spatio-temporal MEG data IEEE Trans. Biomed. Eng. 39 541-57

Tripp J 1983 Physical concepts and mathematical models Biomagnetism: An Interdisciplinary Approach ed S J Williamson, G L Romani, L Kaufman and I Modena (New York: Plenum) pp 101-39

Wang J Z, Williamson S J and Kaufman L 1992 Magnetic source images determined by a lead-field analysis: the unique minimum-norm least-squares estimation IEEE Trans. Biomed. Eng. 39 665-75

Wilson F N and Bayley R H 1950 The electric field of an eccentric dipole in a homogeneous spherical conducting medium Circulation $184-92$

Yvert B, Crouzeix J and Pernier J 2000 High-speed realistic modelling using lead-field interpolation Biomag 2000: Proc. 12th Int. Conf. on Biomagnetism (Espoo, Finland, 13-17 August 2000) at press

Zhang Z 1995 A fast method to compute surface potentials generated by dipoles within multilayer anisotropic spheres Phys. Med. Biol. 40 335-49 